\title{
WEAK AND CLASSICAL SOLUTIONS OF SOME NONLINEAR VOLTERRA INTEGRODIFFERENTIAL EQUATIONS
}

\author{
By
}

$$
\text { Hong-Ming Yin }
$$

IMA Preprint Series \# 920

February 1992 


\title{
Weak and Classical Solutions of Some Nonlinear Volterra Integrodifferential Equations 1
}

\author{
Hong-Ming Yin \\ Department of Mathematics, University of Toronto \\ Toronto, Ontario, M5S 1A1, Canada
}

\begin{abstract}
This paper deals with the following Volterra integrodifferential equation

$$
\begin{aligned}
& u_{t}-\operatorname{div} \vec{A}\left(x, t, u, u_{x}\right)-a\left(x, t, u, u_{x}\right) \\
& =\int_{0}^{t}\left[\operatorname{div} \vec{B}\left(x, t, \tau, u, u_{x}\right)+b\left(x, t, \tau, u, u_{x}\right)\right] d \tau .
\end{aligned}
$$

We first investigate weak solvability. Under certain structure conditions, the global existence of a weak solution is established by means of the Galerkin method. We then study the classical solvability for the above type of equation with a linear principal part. Using the energy method and exploiting the classical maximum principle, we derive some a priori estimates and prove the existence of a classical solution globally. Finally, a qualitative property of solutions is presented.
\end{abstract}

AMS(MOS) Subject Classification: 45K05.

Key Words and Phrases: Nonlinear integrodifferential equations; Global solvability.

\footnotetext{
${ }^{1}$ This work is partially funded by NSERC Canada
} 


\section{Introduction}

In the study of heat conduction in materials with memory, one often encounters the following Volterra integrodifferential equation of parabolic type (VIDEP) (cf. Nohel [13] and Nunziato [14]):

$$
u_{t}=\nabla[a \nabla u(x, t)]+\int_{0}^{t}[\nabla b(x, t, \tau) \nabla u(x, \tau)] d \tau .
$$

There are also some other physical and engineering problems which can be described by such an equation. As an example, one considers the motion of a viscoelastic material whose stress depends on the history of the motion, the equation can be written in the following form: (cf. Dafermos [4], Greenberg [7], e.g.)

$$
u_{t t}=c u_{x x t}+\sigma\left(u_{x}\right)_{x}
$$

or the more general equation in higher space dimension (linear version):

$$
\frac{\partial^{2} u}{\partial t^{2}}-\frac{\partial}{\partial x_{i}}\left[b_{i j}(x, t) \frac{\partial u}{\partial x_{j}}+a(x, t) u\right]-\frac{\partial^{2}}{\partial t \partial x_{i}}\left[a_{i j} \frac{\partial u}{\partial x_{j}}\right]=f(x, t) .
$$

The equation (1.3) can also be used to model the propagation of sound in a viscous medium (cf. Suveika [16]), where the term with the third order derivative serves as a small viscosity. Obviously, it is the same type of equation as (1.1), if one performs the integration over $[0, t]$ on the equation (1.2) or (1.3).

The above physical models motivate us to investigate general parabolic Volterra integrodifferential equations. These equations have been studied for several decades (see the recent proceeding [3]). A common approach is to set the equation in an abstract form and then the theory of analytic semigroups is applied to establish existence. In [2], Crandall, Londen and Nohel considered the abstract equation

$$
u^{\prime}(t)+B u(t)+\int_{0}^{t} a(t-s) A u(s) d s \ni F(t)
$$

where $A$ and $B$ are maximal monotone operators. Under certain assumptions on $A$ and $B$, a weak solution was obtained. As the application of their theory, the equations

$$
u_{t}-\Delta u+\int_{0}^{t} a(t-s) g(u(x, s)) d s=F(t, x)
$$


and

$$
u_{t}-u_{x x}-\int_{0}^{t} a(t-s)\left(\sigma\left(u_{x}(x, s)\right)_{x} d s=F(t, \dot{x})\right.
$$

were discussed (also see [11]). Heard and Rankin [8]-[9] considered the following problem

$$
\left.u^{\prime}(t)+A u(t)=\int_{0}^{t} \frac{\partial}{\partial x_{i}} g_{i}\left(x, t, s, u, u_{x}\right)\right] d s,
$$

where $A$ is a linear elliptic operator and the function $g$ satisfies a uniform Lipschitz condition. With these assumptions, a global weak solution was established via the semigroup theory. Asymptotic behavior of solutions was studied in [9] (also see Fitzgibon [6]). Other work in this category can be found in [3] and the references therein. The other popular way is to regard the equation (1.2) as a mixed type of equation and to establish existence via the energy method (cf. [1], [4], [7], [16], etc.). However, these previous arguments are hardly be applied to a VIDEP with a nonlinear principal part. Moreover, regularity of the weak solution becomes a challenging question. Recently, the author of [17]-[18] considered the equation:

$$
u_{t}=a\left(x, t, u, u_{x}\right) u_{x x}+\int_{0}^{t} b\left(x, t, \tau, u, u_{x}, u_{x x}\right) d \tau .
$$

With the conditions $a \geq a_{0}$ and

$$
|b(x, t, u, p, r)|+\left|b_{t}(x, t, \tau, u, p, r)\right| \leq C[1+|u|+|p|+|r|]
$$

a classical solution was established globally via the theory of parabolic equations. Moreover, in [19] we derived the classical Schauder and $L_{p}$ estimates for a linear VIDEP, which are similar to those for parabolic equations. As an application, we studied the equation

$$
u_{t}=\frac{\partial}{\partial x_{i}}\left[a_{i}\left(x, t, u, \int_{0}^{t} u d \tau\right) u_{x_{i}}\right]+\int_{0}^{t} \frac{\partial}{\partial x_{i}} b_{i}(x, t, u) d \tau
$$

The global solution in the classical sense was obtained.

The present work is a continuation of the recent paper [19]. In this paper we shall deal with the following equation

$$
\begin{aligned}
& u_{t}-\frac{\partial}{\partial x_{i}} a_{i}\left(x, t, u, u_{x}\right)-a\left(x, t, u, u_{x}\right) \\
& =\int_{0}^{t}\left[\frac{\partial}{\partial x_{i}} b_{i}\left(x, \tau, u, u_{x}\right)+b\left(x, t, \tau, u, u_{x}\right)\right] d \tau
\end{aligned}
$$


subject to appropriate initial and boundary conditions. We shall define weak solutions and prove the existence of a weak solution by applying the Galerkin method. Then we will study classical solvability for the equation with a linear principal term:

$$
u_{t}-\Delta u=\int_{0}^{t}[b(t-\tau) \Delta u] d \tau+f(u)
$$

It is well known that for a parabolic equation

$$
u_{t}=\Delta u+f(u)
$$

the one-sided growth condition

$$
f(u) u \leq C\left[u^{2}+1\right]
$$

implies the global existence of a classical solution. This is, however, not so obvious if an integral term is involved in the equation (1.5). By exploiting the maximum principle, we are able to obtain a similar result as that for a parabolic equation. Moreover, using the energy method, we derive some a priori estimates for a semilinear equation with lower order terms and establish the classical solvability. Finally, we present a certain type of maximum principle which we call the quasi-maximum principle.

\section{Weak Solutions}

Let $T>0$ and $Q_{T}=\Omega \times(0, T]$, where $\Omega$ is an open bounded region in $R^{n}$ with a smooth boundary $S=\partial \Omega$, and $S_{T}=S \times[0, T]$. In this section we study weak solutions of the following problem:

$$
\begin{aligned}
& u_{t}-\frac{\partial}{\partial x_{i}} a_{i}\left(x, t, u, u_{x}\right)-a\left(x, t, u, u_{x}\right) \\
& =\int_{0}^{t}\left[\frac{\partial}{\partial x_{i}} b_{i}\left(x, t, \tau, u, u_{x}\right)+b\left(x, t, \tau, u, u_{x}\right)\right] d \tau,(x, t) \in Q_{T}, \\
& u(x, t)=g(x, t), \quad(x, t) \in S_{T}, \\
& u(x, 0)=u_{0}(x), \quad x \in \Omega .
\end{aligned}
$$

Without loss of generality, we may assume that $g(x, t)=0$. We list some structure conditions for the equation. 
$\mathrm{H}(1)$ : The functions $a_{i}, a$ and $b_{i}, b$ are differentiable with respect to all of their arguments in $Q_{T} \times$ $R \times R^{n}$ and $Q_{T} \times R^{+} \times R \times R^{n}$, respectively.

$\mathrm{H}(2)$ : The following ellipticity assumption and the growth conditions hold:

$$
\begin{aligned}
& \sum_{i=1}^{n}\left[a_{i}\left(x, t, u, p_{1}\right)-a_{i}\left(x, t, u, p_{2}\right)\right]\left(p_{1}-p_{2}\right] \geq a_{0}\left(p_{1}-p_{2}\right)^{2} \\
& \sum_{i=1}^{n}\left[\left|a_{i}(x, t, u, p)\right|+\left|b_{i}(x, t, \tau, u, p)\right|\right]+|a|+|b| \leq A_{0}[1+|u|+|p|] \\
& \sum_{i=1}^{n}\left|b_{i p}(x, t, u, p)\right| \leq B_{0},
\end{aligned}
$$

where $a_{0}, A_{0}$ and $B_{0}$ are positive constants.

$\mathrm{H}(3): u_{0}(x) \in H_{0}^{1}(\Omega)$.

Remark 1: One could relax the growth condition a little as in [10]. However, it is not our attention to state the most general theorem.

Definition: A function $u(x, t)$ in $V_{2}\left(Q_{T}\right)=L^{\infty}\left(0, T ; L^{2}(\Omega)\right) \cap L^{2}\left(0, T ; H_{0}^{1}(\Omega)\right)$ is said to be a weak solution of (2.1)-(2.3), if $u(x, t)$ satisfies

$$
\begin{aligned}
& \int_{0}^{T} \int_{\Omega}\left[-u \phi_{t}+\left(a_{i}+\int_{0}^{t} b_{i} d \tau\right) \phi_{x_{i}}-\left(a+\int_{0}^{t} b d \tau\right) \phi\right] d x d t \\
& =\int_{\Omega} u_{0}(x) \phi(x, 0) d x-\int_{\Omega} u(x, T) \phi(x, T) d x
\end{aligned}
$$

for any $\phi(x, t) \in H^{1}\left(0, T ; H_{0}^{1}(\Omega)\right)$.

We shall use the Galerkin method to show the existence of a weak solution. Let $\psi_{k}(x)$ be the normal fundamental basis of the space $H_{0}^{1}(\Omega)$. For any $f(x), g(x)$ in $L^{2}(\Omega)$, we denote

$$
<f(x), g(x)>=\int_{\Omega} f(x) g(x) d x .
$$

Let

$$
u_{m}(x, t)=\sum_{k=1}^{m} c_{k m}(t) \psi_{k}(x)
$$

where $\left\{c_{k m}(t)\right\}$ is the solution of the system:

$$
\frac{d}{d t} c_{k m}(t)+\sum_{i=1}^{n}<a_{i}\left(x, t, u_{m}, u_{m x}\right)+\int_{0}^{t} b_{i}\left(x, t, \tau, u_{m}, u_{m x}\right) d \tau, \psi_{k x_{i}}>
$$




$$
\begin{aligned}
& -<a\left(x, t, u_{m}, u_{m x}\right)+\int_{0}^{t} b\left(x, t, \tau, u_{m}, u_{m x}\right) d \tau, \psi_{k}>=0 \\
& c_{k m}(0)=<u_{0}(x), \psi_{k}>, \quad k=1,2, \cdots, m .
\end{aligned}
$$

We have the following

Lemma 2.1: The system (2.5)-(2.6) admits a unique solution on $[0, T]$ and the solution satisfies the following uniform estimate:

$$
\sum_{k=1}^{m}\left|c_{k m}(t)\right| \leq C
$$

where $C$ depends only on the known data and the upper bound of $T$.

Proof: Local existence is clear. To show global existence, we only need to derive an a priori bound of $\left\{c_{k m}(t)\right\}$. To this end, we multiply (2.5) by $c_{k m}(t)$ and take the summation on $k$. Noting that $c_{k m}(t)=<u_{m}, \psi_{k}>$, we have

$$
\begin{aligned}
& \frac{d}{d t}\left\|u_{m}(\cdot, t)\right\|_{L^{2}(\Omega)}^{2}+\sum_{i=1}^{n}<a_{i}\left(x, t, u_{m}, u_{m x}\right)+\int_{0}^{t} b_{i}\left(x, t, \tau, u_{m}, u_{m x}\right) d \tau, u_{m x_{i}}> \\
& -<a\left(x, t, u_{m}, u_{m x}\right)+\int_{0}^{t} b\left(x, t, \tau, u_{m}, u_{m x}\right) d \tau, u_{m}>=0 .
\end{aligned}
$$

Using the growth assumptions on $a_{i}, b_{i}, a, b$ and Cauchy's inequality, we obtain

$$
\begin{aligned}
& \frac{d}{d t}\left\|u_{m}(\cdot, t)\right\|_{L^{2}(\Omega)}^{2}+a_{0} \int_{\Omega} u_{m x}^{2} d x \\
& \leq C+\varepsilon \int_{\Omega} u_{m x}^{2} d x+C(\varepsilon) \int_{\Omega} u_{m}^{2} d x+C \int_{0}^{t} \int_{\Omega}\left[u_{m}^{2}+u_{m x}^{2}\right] d x d t .
\end{aligned}
$$

By choosing $\varepsilon$ properly and using Gronwall's inequality, we have

$$
\left\|u_{m}\right\|_{V^{2}\left(Q_{T}\right)} \leq C
$$

Thus, the desired result follows from the identity

$$
\max _{0 \leq t \leq T} \sum_{k=1}^{m} c_{k m}^{2}(t)=\max _{0 \leq t \leq T}\left\|u_{m}\right\|_{L^{2}(\Omega)}^{2} \leq\left\|u_{m}\right\|_{V^{2}\left(Q_{T}\right)} .
$$

By the compactnes results (cf. [15]), we see that (a subsequence if necessary)

$$
u_{m}(x, t) \rightarrow u(x, t) \text { in } L^{2}\left(Q_{T}\right) \text { strongly, a.e. in } Q_{T} \text {, and } u_{m x_{i}} \rightarrow u_{x_{i}}(x, t) \text { weakly in } L_{2}\left(Q_{T}\right) \text {. }
$$

Moreover, the weak compactness of $L^{2}\left(Q_{T}\right)$ implies 
$a_{i}\left(x, t, u_{m}, u_{m x}\right), b_{i}\left(x, t, \tau, u_{m}, u_{m x}\right), a\left(x, t, u_{m}, u_{m x}\right), b\left(x, t, \tau, u_{m}, u_{m x}\right)$

are also convergent weakly in $L^{2}\left(Q_{T}\right)$ to certain functions

$A_{i}(x, t), B_{i}(x, t, \tau), A(x, t), B(x, t, \tau)$, respectively, $i=1, \cdots, n$.

Let $\phi(x, t) \in H^{1}\left(0, T ; H_{0}^{1}(\Omega)\right)$, then

$$
\phi(x, t)=\sum_{j=1}^{\infty} \beta_{j}(t) \psi_{j}(x)
$$

We multiply (2.5) by $\beta_{j}(t)$ and then sum over $j$. After integrating over $[0, T]$ and taking limits, we have

$$
\begin{aligned}
& \int_{0}^{T} \int_{\Omega}\left[-u \phi_{t}+\left(A_{i}(x, t)+\int_{0}^{t} B_{i} d \tau\right) \phi_{x_{i}}-\left(A(x, t)+\int_{0}^{t} B d \tau\right) \phi\right] d x d t \\
& =\int_{\Omega} u_{0}(x) \phi(x, 0) d x-\int_{\Omega} u(x, T) \phi(x, T) d x .
\end{aligned}
$$

To show that $u(x, t)$ is a weak solution, we need to show

$$
\begin{aligned}
& \int_{0}^{T} \int_{\Omega}\left[A_{i}(x, t)+\int_{0}^{t} B_{i} d \tau\right] \phi_{x_{i}} d x d t=\int_{0}^{T} \int_{\Omega}\left[a_{i}\left(x, t, u, u_{x}\right)+\int_{0}^{t} b_{i} d \tau\right] \phi_{x_{i}} d x d t ; \\
& \int_{0}^{T} \int_{\Omega}\left[A(x, t)+\int_{0}^{t} B d \tau\right] \phi d x d t=\int_{0}^{T} \int_{\Omega}\left[a+\int_{0}^{t} b d \tau\right] \phi d x d t .
\end{aligned}
$$

Lemma 2.2: For any $u(x, t), v(x, t)$ in $V_{2}\left(Q_{T}\right)$, the following inequality holds for $T \leq T_{0}$ :

$$
\begin{aligned}
& \int_{0}^{T} \int_{\Omega}\left[a_{i}\left(x, t, u, u_{x}\right)-a_{i}\left(x, t, u, v_{x}\right)\right]\left(u_{x_{i}}-v_{x_{i}}\right) d x d t \\
& +\int_{0}^{T} \int_{\Omega}\left\{\int_{0}^{t}\left[b_{i}\left(x, t, \tau, u, u_{x}\right)-b_{i}\left(x, t, \tau, u, v_{x}\right)\right] d \tau\right\}\left(u_{x_{i}}-v_{x_{i}}\right) d x d t \geq 0
\end{aligned}
$$

where $T_{0}$ depends only on $a_{0}$ and $B_{0}$.

Proof: The proof is straightforward by employing the assumption $\mathrm{H}(2)$.

From now on, we always restrict $T \leq T_{0}$. We multiply the equation $(2.5)$ by $\beta_{j}(t)$, take the summation on $j$ and then integrate with respect to $t$ over $[0, T]$ to obtain

$$
\begin{aligned}
& \int_{0}^{T} \int_{\Omega}\left\{-u_{m} \phi_{t}+\left[a\left(x, t, u_{m}, u_{m x}\right)+\int_{0}^{t} b_{i}\left(x, t, \tau, u_{m}, u_{m x}\right) d \tau\right] \phi_{x_{i}}\right. \\
& \left.-\left[a\left(x, t, u_{m}, u_{m x}\right)+\int_{0}^{t} b\left(x, t, \tau, u_{m}, u_{m x}\right) d \tau\right] \phi\right\} d x d t \\
& +\int_{\Omega}\left[u_{m}(x, T) \phi(x, T)-u_{0}(x) \phi(x, 0)\right] d x=0
\end{aligned}
$$


By choosing $\phi(x, t)$ properly and using Lemma 2.2 (cf. [10]), we have for any $\eta \in H^{1}\left(0, T ; H_{0}^{1}(\Omega)\right)$ that

$$
\int_{0}^{T} \int_{\Omega}\left\{\left[A_{i}(x, t)-a_{i}\left(x, t, u, \eta_{x}\right)\right]+\int_{0}^{t}\left[B_{i}(x, t, \tau)-b_{i}\left(x, t, \tau, u, \eta_{x}\right)\right] d \tau\right\}(u-\eta)_{x_{i}} d x d t \geq 0 .
$$

It follows by taking $\eta(x, t)=u(x, t)-\delta \xi(x, t)$ for any $\xi(x, t) \in H^{1}\left(0, T ; H_{0}^{1}(\Omega)\right)$ (see Remark 2 below) and then letting $\delta \rightarrow 0$ that

$$
\int_{0}^{T} \int_{\Omega}\left\{\left[A_{i}(x, t)-a_{i}\left(x, t, u, u_{x}\right)\right]+\int_{0}^{t}\left[B_{i}(x, t, \tau)-b_{i}\left(x, t, \tau, u, u_{x}\right)\right] d \tau\right\} \xi_{x_{i}} d x d t \geq 0 .
$$

This implies

$$
\begin{aligned}
& \int_{0}^{T} \int_{\Omega}\left[A_{i}(x, t)+\int_{0}^{t} B_{i}(x, t, \tau) d \tau\right] \xi_{x_{i}} d x d t \\
& =\int_{0}^{T} \int_{\Omega}\left[a_{i}\left(x, t, u, u_{x}\right)+\int_{0}^{t} b_{i}\left(x, t, \tau, u, u_{x}\right) d \tau\right] \xi_{x_{i}} d x d t .
\end{aligned}
$$

Remark 2: In the above derivation, although $u(x, t)$ does not belong to $H^{1}\left(0, T ; H_{0}^{1}(\Omega)\right)$, one can use the well-known Steklov averging $u_{h}(x, t)$ (cf. [10]) to approximate $u(x, t)$ and then take the limit. With the above equality and the strong convexity condition $\mathrm{H}(2)$, one can actually show that $u_{m x_{i}}(x, t)$ converges to $u_{x_{i}}$ in $L^{2}\left(Q_{T}\right)$ strongly. Indeed, if we choose $\phi(x, t)$ in $(2.8)$ to be $u_{m}(x, t)$ and $u(x, t)$, respectively, noting that $u_{m}(x, t)$ converges $u(x, t)$ in $L^{2}\left(Q_{T}\right)$ strongly, we see

$$
\lim _{m \rightarrow \infty} \int_{0}^{T} \int_{\Omega}\left[a\left(x, t, u_{m}, u_{m x}\right)+\int_{0}^{t} b_{i}\left(x, t, \tau, u_{m}, u_{m x}\right) d \tau\right]\left[u_{m x_{i}}-u_{x_{i}}\right] d x d t=0 .
$$

On the other hand, the weak convergence implies

$$
\lim _{m \rightarrow \infty} \int_{0}^{T} \int_{\Omega}\left[a\left(x, t, u, u_{x}\right)+\int_{0}^{t} b_{i}\left(x, t, \tau, u, u_{x}\right) d \tau\right]\left[u_{m x_{i}}-u_{x_{i}}\right] d x d t=0 .
$$

It follows by $(2.9)$ and $(2.10)$ that

$$
\lim _{m \rightarrow \infty} \int_{0}^{T} \int_{\Omega}\left[I_{i}+J_{i}\right]\left[u_{m x_{i}}-u_{x_{i}}\right] d x d t=0
$$

where

$$
\begin{aligned}
I_{i} & =a_{i}\left(x, t, u_{m}, u_{m x}\right)-a_{i}\left(x, t, u, u_{x}\right) \\
J_{i} & =\int_{0}^{t}\left[b_{i}\left(x, t, \tau, u_{m}, u_{m x}\right)-b_{i}\left(x, t, \tau, u, u_{x}\right)\right] d \tau .
\end{aligned}
$$


We split $I_{i}$ into two terms:

$$
\begin{aligned}
I_{i} & =\left[a\left(x, t, u_{m}, u_{m x}\right)-a_{i}\left(x, t, u_{m}, u_{x}\right)\right]+\left[a_{i}\left(x, t, u_{m}, u_{x}\right)-a_{i}\left(x, t, u, u_{x}\right)\right] \\
& \equiv I_{i}^{*}+I_{i}^{* *} .
\end{aligned}
$$

The strong convergence of $u_{m}(x, t)$ implies

$$
\int_{0}^{T} \int_{\Omega} I_{i}^{* *}\left[u_{m x_{i}}-u_{x_{i}}\right] d x \rightarrow 0
$$

as $m$ tends to $\infty$. The assumption $\mathrm{H}(2)$ yields

$$
\int_{0} \int_{\Omega} I_{i}^{*}\left[u_{m x_{i}}-u_{x_{i}}\right] d x d t \geq a_{0} \int_{0}^{T} \int_{\Omega}\left(u_{m x_{i}}-u_{x_{i}}\right)^{2} d x d t
$$

Similarly, we split $J_{i}$ into two terms $J_{i}=J_{i}^{*}+J_{i}^{* *}$ and have

$$
\int_{0}^{T} \int_{\Omega} J_{i}^{* *}\left[u_{m x_{i}}-u_{x_{i}}\right] d x d t \rightarrow 0
$$

as $m$ tends to $\infty$.

By the Cauchy-Schwartz inequality and $\mathrm{H}(2)$, we see

$$
\begin{aligned}
& \int_{0}^{T} \int_{\Omega}\left|J_{i}^{*}\right|\left|u_{m x_{i}}-u_{x_{i}}\right| d x d t \\
& \leq \delta \int_{0}^{T} \int_{\Omega}\left(u_{m x_{i}}-u_{x_{i}}\right)^{2} d x d t+C(\delta) T \int_{0}^{T} \int_{\Omega}\left[u_{m x_{i}}-u_{x_{i}}\right]^{2} d x d t .
\end{aligned}
$$

It follows that

$$
\left[a_{0}-\delta-C(\delta) T\right] \int_{0}^{T} \int_{\Omega}\left(u_{m x_{i}}-u_{x_{i}}\right)^{2} d x d t \leq \int_{0}^{T} \int_{\Omega}\left[I_{i}^{* *}+J_{i}^{* *}\right]\left[u_{m x_{i}}-u_{x_{i}}\right] d x d t \rightarrow 0
$$

as $m$ approaches $\infty$. By choosing $\delta=\frac{a_{0}}{4}$ and restricting $T \leq T_{0}=\frac{a_{0}}{4 C(\delta)}$ we see that $u_{m x_{i}}(x, t)$ converges to $u_{x_{i}}$ in $L^{2}\left(Q_{T}\right)$ strongly. Consequently, the second equality follows immediately.

Theorem 2.1: Under the hypotheses $\mathrm{H}(1)-\mathrm{H}(3)$, the problem (2.1)-(2.3) admits a unique weak solution.

Proof: The uniqueness can be proved by the standard energy method. We have shown the existence of a weak solution on the interval $\left[0, T_{0}\right]$. However, since $T_{0}$ depends only on $a_{0}$ and $B_{0}$ we can repeat the above procedure and obtain a solution on $\left[T_{0}, 2 T_{0}\right]$. After a finite number of steps, we can obtain a weak solution on the whole interval $[0, T]$ for any $T>0$. 


\section{Classical Solutions}

In this section we consider the following initial-boundary value problem:

$$
\begin{array}{ll}
u_{t}-\Delta u-f(u)= & \int_{0}^{t}[b(t, \tau) \Delta u] d \tau,(x, t) \in Q_{T}, \\
u(x, t)=0, \quad(x, t) \in S_{T}, \quad u(x, 0)=u_{0}(x), & x \in \Omega .
\end{array}
$$

We shall show the global existence of a classical solution to (3.1)-(3.2) under the assumption of a one-sided growth condition which is a little stronger than (1.6). The argument is to exploit the classical maximum principle.

$H^{*}(1)$ : The function $b(t, \tau)$ is differentiable on $R^{2}$;

$H^{*}(2): f(u)$ is differentiable and there exist positive constants $A_{0}$ and $M$ such that

$$
f^{\prime}(u) \leq A_{0}, \text { for }|u| \geq M
$$

$H^{*}(3): u_{0}(x) \in C^{2+\alpha}(\bar{\Omega})$ and the following compatibility conditions hold:

$$
u_{0}(x)=0, \Delta u_{0}(x)+f\left(u_{0}\right)=0 \text { on } S .
$$

Theorem 2: Under the conditions $H^{*}(1)-H^{*}(3)$, the problem (3.1)-(3.2) admits a unique classical solution on $[0, T]$ for any $T>0$.

The proof of local existence is standard. To obtain the global existence, we only need to derive an a priori estimate in $C^{2+\alpha, 1+\frac{\alpha}{2}}\left(\bar{Q}_{T}\right)$. Throughout this section various constants will be denoted by the same symbol $C$, it may be different from one line to the next. Their dependency will be specified in the text.

Lemma 3.1 There exists a constant $C$ which depends on $u_{0}, \Omega$ and the upper bound of $T$ such that

$$
\|u\|_{L^{\infty}\left(Q_{T}\right)} \leq C
$$

Proof: To prove the result, we introduce a new function:

$$
w(x, t)=u(x, t)+\int_{0}^{t} b(t, \tau) u(x, \tau) d \tau,(x, t) \in \bar{Q}_{T} .
$$

Then using the Neumann series, we see that there exists a small number $T_{0}>0$ such that for $t \in\left[0, T_{0}\right]$

$$
u(x, t)=w(x, t)+\int_{0}^{t} B(t, \tau) w(x, \tau) d \tau
$$


where $B(t, \tau)$ is a smooth function and $T_{0}$ depends only on the function $b(t, \tau)$.

It is easy to see that $w(x, t)$ satisfies

$$
w_{t}-\Delta w+B(t, t) w-f(u)=\int_{0}^{t}\left[-B_{t}(t, \tau) w\right] d \tau, \quad(x, t) \in Q_{T_{0}} .
$$

Moreover, $w(x, t)=0$ on $S_{T_{0}}$ and $w(x, 0)=u_{0}(x)$ for $x \in \Omega$.

Let $w(x, t)=e^{\beta t} V(x, t)$, the elementary calculation gives

$$
V_{t}-\Delta V=[-B(t, t)-\beta] V-\int_{0}^{t} B_{t}(t, \tau) e^{-\beta(t-\tau)} V d \tau+f(u) e^{-\beta t} .
$$

Now

$$
\begin{aligned}
f(u) e^{-\beta t} & =\left[\int_{0}^{1} f^{\prime}(\theta u) d \theta u+f(0)\right] e^{-\beta t} \\
& =\int_{0}^{1} f^{\prime}(\theta u) d \theta\left[V(x, t)+\int_{0}^{t} B(t, \tau) e^{-\beta(t-\tau)} V d \tau\right]+f(0) e^{-\beta t} .
\end{aligned}
$$

Now we require that $T_{0}$ is so small such that $\int_{0}^{t} B(t, \tau) d \tau \leq \frac{1}{2}$ for $t \in\left[0, T_{0}\right]$. Assume that $V(x, t)$ attains its positive maximum which is greater than $M$ in $Q_{T_{0}}$. Then at this extreme point, say $\left(x_{0}, t_{0}\right), V\left(x_{0}, t_{0}\right)=M^{*}$ and

$$
V_{t}-\Delta V \geq 0
$$

Moreover,

$$
V\left(x_{0}, t_{0}\right)+\int_{0}^{t_{0}} B\left(t_{0}, \tau\right) e^{-\beta\left(t_{0}-\tau\right)} V\left(x_{0}, \tau\right) d \tau \geq M^{*}-\frac{1}{2} M^{*}>0 .
$$

The condition $H^{*}(2)$ implies

$$
\begin{aligned}
& \int_{0}^{1} f^{\prime}(\theta u) d \theta\left[V\left(x_{0}, t_{0}\right)+\int_{0}^{t_{0}} B\left(t_{0}, \tau\right) e^{-\beta\left(t_{0}-\tau\right)} V\left(x_{0}, \tau\right) d \tau\right] \\
& \leq A_{0}\left[V\left(x_{0}, t_{0}\right)+\int_{0}^{t_{0}} B\left(t_{0}, \tau\right) e^{-\beta\left(t_{0}-\tau\right)} V\left(x_{0}, \tau\right) d \tau\right] \\
& \leq A_{0}\left[M^{*}+B_{0} T_{0} M^{*}\right]
\end{aligned}
$$

where

$$
B_{0}=\max _{[0, T] \times[0, T]}|B(t, \tau)| .
$$

It follows by combining the above inequalities that at $\left(x_{0}, t_{0}\right)$

$$
\left[\beta+B\left(t_{0}, t_{0}\right)-A_{0}-B_{0} T_{0}\right] M^{*} \leq \max \left\{M, f(0) e^{-\beta t_{0}}\right\} .
$$


Consequently, we have

$$
M^{*} \leq 2 \max \left\{M, f(0) e^{-\beta T}\right\}
$$

if we choose $\beta+B(t ; t)-A_{0}-B_{0} T_{0} \geq \frac{1}{2}$.

This yields

$$
\max _{Q_{T}} V(x, t) \leq \max \left\{\max _{\Omega}\left|u_{0}(x)\right|, 2 M, 2 f(0) e^{-\beta T}\right\} .
$$

Similarily, we can obtain the lower bound. Finally, since $T_{0}$ depends only on $b(t, \tau)$, we can repeat the above procedure to obtain the above estimate on $\left[T_{0}, 2 T_{0}\right]$. After a finite number of steps, we can obtain the estimate $(3.6)$ on $[0, T]$ for any fixed $T>0$. Thus, the desired estimate in Lemma 3.1 follows from the relation (3.4).

Lemma 3.2 : There exists a constant $C$ with the same dependency as in Lemma 3.1 such that

$$
\|u\|_{C^{2+\alpha, 1+\frac{\alpha}{2}\left(\bar{Q}_{T}\right)}} \leq C .
$$

Proof: To show estimates, we employ the $W_{p}^{2,1}\left(Q_{T}\right)$-estimate (cf. [19]) for any $p>1$ to yield

$$
\|u\|_{W_{p}^{2,1}\left(Q_{T}\right)} \leq C
$$

The interpolation inequality implies

$$
\|u\|_{C^{1+\alpha} \frac{1+\alpha}{2}\left(\bar{Q}_{T}\right)} \leq C .
$$

Finally, the Schauder estimate (cf. [19]) infers

$$
\|u\|_{C^{2+\alpha, 1+\frac{\alpha}{2}\left(\bar{Q}_{T}\right)}} \leq C
$$

where the constant $C$ depends only on the known data, $\Omega$ and the upper bound of $T$.

With the above a priori estimate at hand, we can use the method of continuation to establish the existence of a classical solution on the whole interval $[0, T]$ for any given $T>0$ (cf. [19]). Hence we obtain Theorem 2 .

The proof of Lemma 3.1 is based on the classical maximum principle. Here we present a proof for a particular nonlinear function, namely,

$$
f(u)=u-u^{2 m+1}
$$


where $m$ is a positive integer. The method relies on various energy estimates instead of using the maximum principle, although $H^{*}(2)$ holds for this $f(u)$. This argument allows us to deal with a more general class of semilinear integrodifferential equations.

Lemma 3.3: For the above particular $f(u)$, the estimate (3.3) holds.

Proof: It is easy to see that

$$
\int_{\Omega} u^{2} d x+\int_{0}^{t} \int_{\Omega} u_{x}^{2} d x d t \leq C
$$

As in the proof of Lemma 3.2, let

$$
w(x, t)=u(x, t)+\int_{0}^{t} b(t, \tau) u(x, \tau) d \tau
$$

Then $w(x, t)$ satisfies

$$
w_{t}-\triangle w+B(t, t) w=\int_{0}^{t}\left[-B_{t}(t, \tau)\right] w d \tau+f(u)
$$

We calculate the following integral for $p \geq 2$ even:

$$
\begin{aligned}
& \int_{0}^{T} \frac{d}{d t} \int_{\Omega} w^{p} d x d t=\int_{0}^{t} \int_{\Omega} p w^{p-1} w_{t} d x d t \\
& =\int_{0}^{T} p w^{p-1}\left\{[\Delta w-B(t, t) w+f(u)]+\int_{0}^{t}\left[-B_{t}(t, \tau) w d\right] \tau\right\} d x d t
\end{aligned}
$$

After executing the integration by parts, we have

$$
\begin{aligned}
& \int_{\Omega} w(x, T)^{p} d x+p(p-1) \int_{0}^{T} \int_{\Omega} w^{p-2} w_{x}^{2} d x d t \\
& \leq \int_{\Omega} u_{0}(x)^{p} d x+I
\end{aligned}
$$

where

$$
I=\int_{0}^{T} \int_{\Omega} p w^{p-1}\left[-B(t, t) w+f(u)+\int_{0}^{t}\left[-B_{t}(t, \tau) w\right] d \tau\right] d x d t .
$$

Now we split $I$ into three terms denoted by $I_{1}, I_{2}$ and $I_{3}$, respectively. By using the Hölder and Young inequalities, we see

$$
\begin{gathered}
\left|I_{1}\right|+\left|I_{3}\right| \leq C p \int_{0}^{T} \int_{\Omega} w^{p} d x d t \\
I_{2}=\int_{0}^{T} \int_{\Omega} p w^{p-1}\left[u-u^{2 m+1}\right] d x d t \equiv J_{1}+J_{2} .
\end{gathered}
$$


It is clear that

$$
\begin{gathered}
\left|J_{1}\right| \leq C \int_{0}^{T} \int_{\Omega} p w^{p} d x d t \\
J_{2}=-\int_{0}^{T} \int_{\Omega} p w^{p-1}\left[w+\int_{0}^{t} B(t, \tau) w d \tau\right]^{2 m+1} d x d t \\
=-\int_{0}^{T} \int_{\Omega} p w^{p-1}\left\{w^{2 m+1}+\sum_{i=0}^{2 m}\left({ }_{i}^{2 m+1}\right) w^{i}\left[\int_{0}^{t} B(t, \tau) w d \tau\right]^{2 m+1-i}\right\} d x d t \\
\left.=-\int_{0}^{T} \int_{\Omega} p w^{p+2 m} d x d t-\int_{0}^{T} \int_{\Omega} p w^{p-1} \sum_{i=0}^{2 m}\left({ }_{i}^{2 m+1}\right) w^{i}\left[\int_{0}^{t} B(t, \tau) u d \tau\right]^{2 m+1-i}\right\} d x d t \\
=K_{1}+K_{2} .
\end{gathered}
$$

We use the Hölder and Young inequalities to obtain

$$
\left|K_{2}\right| \leq \delta \int_{0}^{T} \int_{\Omega} p w^{p+2 m} d x d t+C(\delta) T \int_{0}^{T} \int_{\Omega} p w^{p+2 m} d x d t .
$$

However, both terms on the right-hand side of the above inequality can be dominated by $K_{1}$, by restricting $\delta$ and $T \leq T_{0}$ to be small enough. Therefore, in a small interval $\left[0, T_{0}\right]$, we have

$$
\begin{aligned}
& \int_{\Omega} w(x, T)^{p} d x+p(p-1) \int_{0}^{T} \int_{\Omega} w^{p-2} w_{x}^{2} d x d t \\
& \leq \int_{\Omega} u_{0}(x)^{p} d x+C p \int_{0}^{T} \int_{\Omega} w^{p} d x d t .
\end{aligned}
$$

Using the iteration technique (cf. [19]), we have

$$
\|w\|_{L^{\infty}\left(Q_{T}\right)} \leq C
$$

As $T_{0}$ depends only on the known data, we can repeat the process to achieve the desired estimate in the whole interval $[0, T]$ for any given $T>0$. Thus, we have

Theorem 3: Assume that $f(u)=u-u^{2 m+1}$ and the other conditions in Theorem 2 , then the problem (3.1)-(3.2) admits a unique classical solution globally.

Remark 3: The energy method can be used to deal with the following more general equation:

$$
u_{t}-\Delta u+a\left(x, t, u, u_{x}\right)=\int_{0}^{t}\left[B(t, \tau) \triangle u d+\frac{\partial}{\partial x_{i}} b_{i}(x, t, u)+c(x, t, \tau, u)\right] \tau+f(u),
$$

where the functions $a(x, t, u, p), b_{i}(x, t, \tau, u)$ and $c(x, t, \tau, u)$ satisfiy the joint linear growth condition

$$
|a|+|b|+|c| \leq C[1+|u|+|p|]
$$


and $f(u)$ is the same as in Theorem 3. Then we have the global existence for the above equation. We omit to state the result here (cf.[19]).

Remark 4: It would be very interesting to use the energy method ; instead of the maximum principle, to derive the maximum norm of solutions for general $f(u)$ with one-sided condition (1.6).

\section{Quasi-maximum Principle}

In this section we prove a theorem which provides an a priori bound of solutions of a semilinear equation. More specifically, we consider the following equation

$$
u_{t}=\Delta u+\int_{0}^{t} b(t, \tau) \Delta u d t+c(x, t) u,(x, t) \in Q_{T}
$$

subject to the initial and boundary conditions:

$$
\begin{aligned}
u(x, t)=g(x, t), & (x, t) \in S_{T}, \\
u(x, 0)=u_{0}(x), & (x, t) \in \Omega .
\end{aligned}
$$

It is easy to see the classical maximum principle can not be directly applied in general. However, we have seen that a modified principle still holds.

Theorem 4.1: (Quasi-maximum Principle) Assume that $c(x, t) \leq C_{0}$, then there exists a constant $K>0$ which depends only on $C_{0}$ and $b(t, \tau)$ such that

$$
e^{K T} \min \left\{\min _{S_{T}} G(x, t), \min _{\bar{\Omega}} u_{0}(x)\right\} \leq w(x, t) \leq e^{K T} \max \left\{\max _{S_{T}} G(x, t), \max _{\bar{\Omega}} u_{0}(x)\right\},
$$

where $w(x, t)=u(x, t)+\int_{0}^{t} b(t, \tau) u d \tau$ and $G(x, t)=g(x, t)+\int_{0}^{t} b(t, \tau) g(x, \tau) d \tau$.

Proof: As in Section 3,w(x,t) satisfies

$$
w_{t}=\Delta w+[c(x, t)-B(t, t)] w+\int_{0}^{t}\left[c(x, t) B(t, \tau)-B_{t}(t, \tau)\right] w d \tau
$$

Let $V(x, t)=e^{-K t} w$, where $K$ is a large positive constant. For the equation of $V$, we are able to use the classical maximum principle to obtain the desired result.

Acknowledgement: After this paper was completed, the author had an opportunity to discuss the results with a number of colleagues, in particular, Professor J. Nohel, Professor H. Engler and Professor S. Rankin III. He would like to thank them for their interest in the results. The author also thanks the referee for some helpful comments. He wants to thank Prof. G. Duff for his careful reading the manuscript. 


\section{References}

1. G. Andrews, On existence of solutions to the equation $u_{t t}=u_{x x t}+\sigma\left(u_{x}\right)_{x}$, J. Diff. Eqs., $35(1980), 200-231$.

2. M.G. Crandall, S.O. Londen and J.A. Nohel, An abstract nonlinear Volterra integrodifferential equation, J. Math. Anal. Appl., 64(1978), 701-735.

3. G. Da Prato and M. Iannelli, Volterra Integrodifferential Equations in Banach Sapces and Applications, Pitman Research Notes in Math. Series, Vol. 190, Longman, UK.

4. C. M. Dafermos, The mixed initial boundary value problem for the equations of nonlinear one dimensional visco-elasticity, J. Diff. Eqs., 6(1969), 71-86.

5. H. Engler, Global regular solutions for the dynamic antiplane shear problem in nonlinear viscoelasticity, Math. Z. 202(1989), 251-259.

6. W.E. Fitzgibbon, Asymptotic behavior of solutions to a class of Volterra integrodifferential equations, J. of Math. Anal. and Appl., 146(1990), 241-253.

7. J. M. Greenberg, On the existence, uniqueness and stability of the equation $\rho_{0} X_{t t}=E\left(X_{x}\right) X_{x x}+X_{x x t}$, J. MAth. Anal. Appl., 25(1969), 575-591.

8. M. L. Heard and S.M. Rankin III, A semilinear parabolic Volterra integrodifferential equation, J. Diff. Eqs., 71(1988), 201-233.

9. M.L. Heard and S.M. Rankin III, Weak solutions for a class of parabolic Volterra integrodifferential equations, J. of Math. Anal. and Appl., 139(1989), 78-109.

10. O.A. Ladyzenskaja, V.A. Solonnikov and N.N. Ural'ceva, "Linear and quasi-linear equations of parabolic type," Amer. Math. Soc. Trans, vol. 23, Providence, R.I. (1968).

11. S.O. Londen and J. A. Nohel, Nonlinear Volterra integrodifferential equation occurring in heat flow, Journal of Integral Eqs., 6(1984), 11-50.

12. A. Lunardi and E. Sinestrari, Fully Nonlinear Integrodifferential Equations in general Banach spaces, Math. Z., 190(1985), 225-248. 
13. J.A. Nohel, Nonlinear Volterra equations for heat flow in materials with memory, Integral and functional diff. eqs., Lecture notes in pure and applied math., ed. by T.L. Herdman, S.M. Rankin, III, H.W. Stech, Marcel Dekker Inc.,1981.

14. J. W. Nunziato, On heat equation in materials with memory, Quarterly of Applied Math., (1971), 187-204.

15. J. Simon, Compact sets in the space $L^{p}(0, T ; B)$, Anali Mat. Pura Appl., 146(1987),65-96.

16. I.V. Suveika, Mixed problems for an equation describing the propagation od disturbances in viscous media, Diff. Eqs., 19(1984), 337-347.

17. H.M. Yin, The classical solutions for nonlinear integrodifferential equations, J. Integral Equations and Applications, 1(1988), 249-263.

18. H.M. Yin and Y. Lin, Existence for one-dimensional nonlinear parabolic Volterra integrodifferential equations, Submitted.

19. H.M. Yin, On Volterra integrodifferential equations in Several space dimensions, SIAM Journal on Mathematical Analysis, 22(1991), 1723-1737. 
839 Oscar P. Bruno and Fernando Reitich, Numerical solution of diffraction problems: a method of variation of boundaries

840 Oscar P. Bruno and Fernando Reitich, Solution of a boundary value problem for Helmholtz equation via variation of the boundary into the complex domain

841 Victor A. Galaktionov and Juan L. Vazquez, Asymptotic behaviour for an equation of superslow diffusion. The Cauchy problem

Josephus Hulshof and Juan Luis Vazquez, The Dipole solution for the porous medium equation in several space dimensions

843 Shoshana Kamin and Juan Luis Vazquez, The propagation of turbulent bursts

844 Miguel Escobedo, Juan Luis Vazquez and Enrike Zuazua, Source-type solutions and asymptotic behaviour for a diffusion-convection equation

Marco Biroli and Umberto Mosco, Discontinuous media and Dirichlet forms of diffusion type

Stathis Filippas and Jong-Shenq Guo, Quenching profiles for one-dimensional semilinear heat equations

H. Scott Dumas, A Nekhoroshev-like theory of classical particle channeling in perfect crystals

R. Natalini and A. Tesei, On a class of perturbed conservation laws

Paul K. Newton and Shinya Watanabe, The geometry of nonlinear Schrödinger standing waves

S.S. Sritharan, On the nonsmooth verification technique for the dynamic programming of viscous flow

Mario Taboada and Yuncheng You, Global attractor, inertial manifolds and stabilization of nonlinear damped beam equations

Shigeru Sakaguchi, Critical points of solutions to the obstacle problem in the plane

854

855

856

F. Abergel, D. Hilhorst and F. Issard-Roch, On a dissolution-growth problem with surface tension in the neighborhood of a stationary solution

Erasmus Langer, Numerical simulation of MOS transistors

Hä̈m Brezis and Shoshana Kamin, Sublinear elliptic equations in $\mathbf{R}^{n}$

Johannes C.C. Nitsche, Boundary value problems for variational integrals involving surface curvatures

Chao-Nien Chen, Multiple solutions for a semilinear elliptic equation on $\mathbf{R}^{N}$ with nonlinear dependence on the gradient

D. Brochet, X. Chen and D. Hilhorst, Finite dimensional exponential atttractor for the phase field model

Joseph D. Fehribach, Mullins-Sekerka stability analysis for melting-freezing waves in helium-4

Walter Schempp, Quantum holography and neurocomputer architectures

D.V. Anosov, An introduction to Hilbert's 21st problem

Herbert E Huppert and M Grae Worster, Vigorous motions in magma chambers and lava lakes

Robert L. Pego and Michael I. Weinstein, A class of eigenvalue problems, with applications to instability of solitary waves

Mahmoud Affouf, Numerical study of a singular system of conservation laws arising in enhanced oil reservoirs

Darin Beigie, Anthony Leonard and Stephen Wiggins, The dynamics associated with the chaotic tangles of two dimensional quasiperiodic vector fields: theory and applications

Gui-Qiang Chen and Tai-Ping Liu, Zero relaxation and dissipation limits for hyperbolic conservation laws

Gui-Qiang Chen and Jian-Guo Liu, Convergence of second-order schemes for isentropic gas dynamics

Aleksander M. Simon and Zbigniew J. Grzywna, On the Larché-Cahn theory for stress-induced diffusion

Jerzy Luczka, Adam Gadomski and Zbigniew J. Grzywna, Growth driven by diffusion

Mitchell Luskin and Tsorng-Whay Pan, Nonplanar shear flows for nonaligning nematic liquid crystals

Mahmoud Affouf, Unique global solutions of initial-boundary value problems for thermodynamic phase transitions

Richard A. Brualdi and Keith L. Chavey, Rectangular $L$-matrices

Xinfu Chen, Avner Friedman and Bei Hu, The thermistor problem with zero-one conductivity II

Raoul LePage, Controlling a diffusion toward a large goal and the Kelly principle

Raoul LePage, Controlling for optimum growth with time dependent returns

Marc Hallin and Madan L. Puri, Rank tests for time series analysis a survey

V.A. Solonnikov, Solvability of an evolution problem of thermocapillary convection in an infinite time interval

Horia I. Ene and Bogdan Vernescu, Viscosity dependent behaviour of viscoelastic porous media

Kaushik Bhattacharya, Self-accommodation in martensite

D. Lewis, T. Ratiu, J.C. Simo and J.E. Marsden, The heavy top: a geometric treatment

Leonid V. Kalachev, Some applications of asymptotic methods in semiconductor device modeling

David C. Dobson, Phase reconstruction via nonlinear least-squares

Patricio Aviles and Yoshikazu Giga, Minimal currents, geodesics and relaxation of variational integrals on mappings of bounded variation 
Charles R. Johnson and Michael Lundquist, Operator matrices with chordal inverse patterns

Charles M. Elliott and Stefan Luckhaus, 'A generalised diffusion equation for phase separation of a multi-compon mixture with interfacial free energy'

Christian Schmeiser and Andreas Unterreiter, The derivation of analytic device models by asymptotic methods

LeRoy B. Beasley and Norman J. Pullman, Linear operators that strongly preserve the index of imprimitivity

Jerry Donato, The Boltzmann equation with lie and cartan

Thomas R. Hoffend Jr., Peter Smereka and Roger J. Anderson, A method for resolving the laser induced local heating of moving magneto-optical recording media

E.G. Kalnins, Willard Miller, Jr. and Sanchita Mukherjee, Models of $q$-algebra representations: the group of plane motions

T.R. Hoffend Jr. and R.K. Kaul, Relativistic theory of superpotentials for a nonhomogeneous, spatially isotropic medium

Reinhold von Schwerin, Two metal deposition on a microdisk electrode

Vladimir I. Oliker and Nina N. Uraltseva, Evolution of nonparametric surfaces with speed depending on curvature, III. Some remarks on mean curvature and anisotropic flows

Wayne Barrett, Charles R. Johnson, Raphael Loewy and Tamir Shalom, Rank incrementation via diagonal perturbations

Mingxiang Chen, Xu-Yan Chen and Jack K. Hale, Structural stability for time-periodic one-dimensional parabolic equations

Hong-Ming Yin, Global solutions of Maxwell's equations in an electromagnetic field with the temperaturedependent electrical conductivity

Robert Grone, Russell Merris and William Watkins, Laplacian unimodular equivalence of graphs

Miroslav Fiedler, Structure-ranks of matrices

Miroslav Fiedler, An estimate for the nonstochastic eigenvalues of doubly stochastic matrices

Miroslav Fiedler, Remarks on eigenvalues of Hankel matrices

Charles R. Johnson, D.D. Olesky, Michael Tsatsomeros and P. van den Driessche, Spectra with positive elementary symmetric functions

Pierre-Alain Gremaud, Thermal contraction as a free boundary problem

K.L. Cooke, Janos Turi and Gregg Turner, Stabilization of hybrid systems in the presence of feedback delays

Robert P. Gilbert and Yongzhi Xu, A numerical transmutation approach for underwater sound propagation

LeRoy B. Beasley, Richard A. Brualdi and Bryan L. Shader, Combinatorial orthogonality

Richard A. Brualdi and Bryan L. Shader, Strong hall matrices

Håkan Wennerström and David M. Anderson, Difference versus Gaussian curvature energies; monolayer versus bilayer curvature energies applications to vesicle stability

Shmuel Friedland, Eigenvalues of almost skew symmetric matrices and tournament matrices

Avner Friedman, Bei Hu and J.L. Velazquez, A Free Boundary Problem Modeling Loop Dislocations in Crystals

Ezio Venturino, The Influence of Diseases on Lotka-Volterra Systems

Steve Kirkland and Bryan L. Shader, On Multipartite Tournament Matrices with Constant Team Size

Richard A. Brualdi and Jennifer J.Q. Massey, More on Structure-Ranks of Matrices

Douglas B. Meade, Qualitative Analysis of an Epidemic Model with Directed Dispersion

Kazuo Murota, Mixed Matrices Irreducibility and Decomposition

Richard A. Brualdi and Jennifer J.Q. Massey, Some Applications of Elementary Linear Algebra in Combinations

Carl D. Meyer, Sensitivity of Markov Chains

Hong-Ming Yin, Weak and Classical Solutions of Some Nonlinear Volterra Integrodifferential Equations

B. Leinkuhler and A. Ruehli, Exploiting Symmetry and Regularity in Waveform Relaxation Convergence Estimation

Xinfu Chen and Charles M. Elliott, Asymptotics for a Parabolic Double Obstacle Problem

Yongzhi Xu and Yi Yan, An Approximate Boundary Integral Method for Acoustic Scattering in Shallow Oceans Yongzhi Xu and Yi Yan, Source Localization Processing in Perturbed Waveguides

Kenneth L. Cooke and Janos Turi, Stability, Instability in Delay Equations Modeling Human Respiration F. Bethuel, H. Brezis, B.D. Coleman and F. Hélein, Bifurcation Analysis of Minimizing Harmonic Maps Describing the Equilibrium of Nematic Phases Between Cylinders

Frank W. Elliott, Jr., Signed Random Measures: Stochastic Order and Kolmogorov Consistency Conditions D.A. Gregory, S.J. Kirkland and B.L. Shader, Pick's Inequality and Tournaments

J.W. Demmel, N.J. Higham and R.S. Schreiber, Block $L U$ Factorization

Victor A. Galaktionov and Juan L. Vazquez, Regional Blow-Up in a Semilinear Heat Equation with Convergence to a Hamilton-Jacobi Equation

Bryan L. Shader, Convertible, Nearly Decomposable and Nearly Reducible Matrices

Dianne P. O'Leary, Iterative Methods for Finding the Stationary Vector for Markov Chains 